The Journal of $\mathbf{N}_{\text {onlinear }} \mathbf{S}_{\text {ciences and its }}$ A pplications http://www.tjnsa.com

\title{
MULTIPLE POSITIVE SOLUTIONS FOR NONLINEAR SINGULAR THIRD-ORDER BOUNDARY VALUE PROBLEM IN ABSTRACT SPACES
}

\author{
FANG ZHANG ${ }^{1 *}$ \\ Communicated by Professor J. H. He
}

\begin{abstract}
In this paper, we study the nonlinear singular boundary value problem in abstract spaces:

$$
\left\{\begin{array}{l}
u^{\prime \prime \prime}+f(t, u)=\theta, \quad t \in(0,1) \\
u(0)=u^{\prime}(0)=\theta, u^{\prime}(1)=\xi u^{\prime}(\eta)
\end{array}\right.
$$

where $0<\eta<1$ and $1<\xi<\frac{1}{\eta}, \theta$ denotes the zero element of $E, E$ is a real Banach space, and $f(t, u)$ is allowed to be singular at both end point $t=0$ and $t=1$. We show the existence of at least two positive solutions of this problem.
\end{abstract}

\section{INTRODUCTION}

In this paper, we consider the following singular boundary value problem (BVP) for third-order differential equations in a Banach space $E$ :

$$
u^{\prime \prime \prime}(t)+f(t, u(t))=\theta, \quad 0<t<1
$$

subject to the boundary conditions

$$
u(0)=u^{\prime}(0)=\theta, u^{\prime}(1)=\xi u^{\prime}(\eta),
$$

where $0<\eta<1$ and $1<\xi<\frac{1}{\eta}, f \in C[(0,1 / 2) \times P, P]$ which may be singular at $t=0, t=1 ; P$ is a cone of Banach space $E$, which will be stated in detail in section $2 ; \theta$ is the zero element of $E$.

Date: Received: 13 July 2008; Revised: 24 July 2008.

* Corresponding author.

2000 Mathematics Subject Classification. Primary 34G20; Secondary 34B16.

Key words and phrases. Singular boundary value problem, Abstract spaces, Positive solutions, Fixed point theorem. 
Boundary value problems arise from applied mathematics and physics, and they have received a great deal of attention in the literature. Problems of the form (1.1) subject to $(1.2)$, for example, are used to model such phenomena as the deflection of a curved beam having a constant or varying cross section, a threelayer beam, electromagnetic waves or gravity driven flows and so on [1]. Thirdorder boundary value problems have been studied widely in the literature (see [1]-[12] and references therein). However, all of the above-mentioned references consider (1.1) only in scalar space. On the other hand, the theory of ordinary differential equations(ODE) in abstract spaces is becoming an important branch of mathematics in last thirty years because of its application in partial differential equations and ODE's in appropriately infinite dimensional spaces(see, for example [13], [14]). As a result the goal of this paper is to fill up the gap in this area, that is, to investigate the existence of multiple positive solutions of $(1.1)$ with $(1.2)$ in a Banach space $E$.

This paper is organized as follows. Section 2 gives some preliminaries and some lemmas. Section 3 is devoted to the main results and the proof of the results; an example is worked out to indicate the application of our main results.

\section{PRELIMINARIES AND LEMmaS}

In this paper, we suppose throughout that $E$ is a real Banach space. A nonempty closed convex subset $P$ in $E$ is said to be a cone which defined a partial ordering in $E$ by $x \leq y$ if and only if $y-x \in P, P$ is said to be normal if there exists a positive constant $N$ such that $\theta \leq x \leq y$ implies $\|x\| \leq N\|y\|$, where $\theta$ denotes the zero element of $E$, and the smallest $N$ is called the normal constant of $P$ (it is clear, $N \geq 1$ ). For details on cone theory, see [15]. Let $S$ be a bounded subset of a Banach space. $\alpha(S)$ denotes the Kuratowski's measure of noncompactness of $S$. In this paper, $\alpha(\cdot)$ denotes the Kuratowski's measure of noncompactness of a bounded subset of both $E$ and $C[[0,1], E]$. Let

$$
C[[0,1], E]=\{u:[0,1] \rightarrow E \mid u(t) \text { is continuous on }[0,1]\},
$$

$C^{2}[[0,1], E]=\{u:[0,1] \rightarrow E \mid u(t)$ is second order continuously differentiable in $[0,1]\}$, $C^{3}[(0,1), E]=\{u:(0,1) \rightarrow E \mid u(t)$ is third order continuously differentiable in $(0,1)\}$.

For $u=u(t) \in C[[0,1], E]$, let $\|u\|_{C}=\max _{0 \leq t \leq 1}\|u(t)\|$, then $C[[0,1], E]$ becomes a Banach space. Let $Q=\{u \in C[[0,1], E] \mid u(t) \geq \theta, t \in[0,1]\}$, then $Q$ is a cone in $C[[0,1], E]$. An operator $u(t) \in C[[0,1], E] \cap C^{3}[(0,1), E]$ is called a positive solution of the BVP 1.1)-1.2) if $u(t)$ satisfies $1.1-(1.2)$ and $u \in Q, u(t) \not \equiv$ $\theta, t \in[0,1]$.

Lemma 2.1. [12] Let $\xi \eta \neq 1$. Then for $y \in C[[0,1], E]$, the $B V P$ :

$$
\begin{aligned}
& u^{\prime \prime \prime}+y(t)=\theta, \quad t \in(0,1) \\
& u(0)=u^{\prime}(0)=\theta, u^{\prime}(1)=\xi u^{\prime}(\eta),
\end{aligned}
$$

has a unique solution

$$
u(t)=\int_{0}^{1} G(t, s) y(s) d s
$$


where

$$
G(t, s)=\frac{1}{2(1-\xi \eta)}\left\{\begin{array}{r}
\left(2 t s-s^{2}\right)(1-\xi \eta)+t^{2} s(\xi-1), \quad s \leq \min \{\eta, t\}, \\
t^{2}(1-\xi \eta)+t^{2} s(\xi-1), \quad t \leq s \leq \eta, \quad \eta s \leq t, \quad \max \{\eta, t\} \leq s
\end{array}\right.
$$

is called the Green's function.

Lemma 2.2. [12] Let $0<\eta<1$ and $1<\xi<\frac{1}{\eta}$. Then for $G(t, s)$ defined in (2.1), we have estimates

(i) for any $(t, s) \in[0,1] \times[0,1], 0 \leq G(t, s) \leq \Phi(s)$, where

$$
\Phi(s)=\frac{1+\xi}{1-\xi \eta} s(1-s) .
$$

(ii) for any $(t, s) \in\left[\frac{\eta}{\xi}, \eta\right] \times[0,1], G(t, s) \geq \gamma \Phi(s)$, where

$$
0<\gamma=\frac{\eta^{2}}{2 \xi^{2}(1+\xi)} \min \{\xi-1,1\}<1
$$

Let us list some conditions.

$(H 1) f(t, u) \leq g(t) h(u), t \in(0,1), u \in P$, where $g:(0,1) \rightarrow[0, \infty)$ is continuous, and $h: P \rightarrow P$ is continuous and maps bounded subsets to bounded subsets.

(H2) $0<\int_{0}^{1} \Phi(s) g(s) d s<\infty$.

(H3) There exists a $\varphi \in P^{*}$ such that $u>\theta$ implies that $\varphi(u)>0$ and

$$
m_{0}<\lim _{\substack{\|u\| \rightarrow 0 \\ u \in P}} \inf _{\substack{u \in\left[\frac{\eta}{\xi}, \eta\right] \\ \text { ( }}} \frac{\varphi(f(t, u))}{\varphi(u)} \leq \infty,
$$

where $P^{*}=\left\{\varphi \in E^{*} \mid \varphi(u) \geq 0, u \in P\right\}$ denotes the dual cone of $P$, and $m_{0}=$ $\left(\min _{t \in\left[\frac{\eta}{\xi}, \eta\right]} \int_{\frac{\eta}{\xi}}^{\eta} G(t, s) d s\right)^{-1}$.

(H4) There exists a $\varphi_{1} \in P^{*}$ such that $u>\theta$ implies that $\varphi_{1}(u)>0$ and

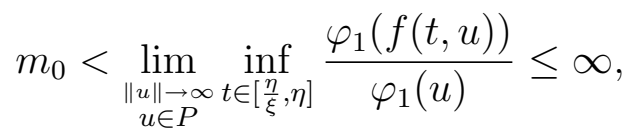

where $P^{*}$ and $m_{0}$ are the same as in $(H 3)$.

(H5) There exists a $\eta_{0}>0$ such that $u \in P,\|u\| \leq \eta_{0}$ implies that

$$
\|h(u)\|<M_{0} \eta_{0}
$$

where $M_{0}=\left(N \int_{0}^{1} \Phi(s) g(s) d s\right)^{-1}$, and $N$ denotes the normal constant of $P$.

(H6) $0 \leq \varlimsup_{\substack{\|u\| \rightarrow 0 \\ u \in P}} \frac{\|h(u)\|}{\|u\|}<M_{0}$, where $M_{0}$ is as in (H5).

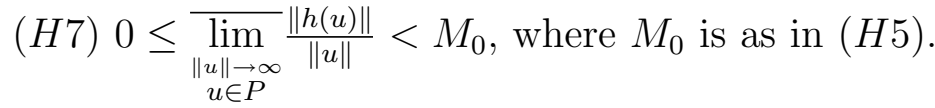


(H8) There exists a $\varphi_{2} \in P^{*}$ such that $u>\theta$ implies that $\varphi_{2}(u)>0$. Then exists a $\eta_{1}>0$ such that $t \in\left[\frac{\eta}{\xi}, \eta\right], u \in P$ and $\frac{\gamma}{N} \eta_{1} \leq\|u\| \leq \eta_{1}$ imply

$$
\varphi_{2}(f(t, u))>m_{0} \varphi_{2}(u)
$$

where $P^{*}$ and $m_{0}$ are the same as in $(H 3)$.

(H9) For $l>0,[a, b] \subset(0,1), f$ is uniformly continuous on $[a, b] \times\left(P \cap T_{l}\right)$, where $T_{l}=\{u \in E \mid\|u\| \leq l\}$, and there exists a constant $L: 0 \leq L<\frac{1}{2 M_{1}}$ such that for $t \in(0,1)$ and bounded subsets $D \subset P$,

$$
\alpha(f(t, D)) \leq L \alpha(D)
$$

holds, where $M_{1}=\max _{s \in[0,1]} \Phi(s)$.

Now we define

$$
(A u)(t)=\int_{0}^{1} G(t, s) f(s, u(s)) d s, \forall u \in Q,
$$

then $(A u)(t) \geq \theta, t \in[0,1]$, and using the Lebesgue dominated convergence theorem we know that $(A u)(t)$ is continuous on $[0,1]$, hence the integral operator $A: Q \rightarrow Q$. Further, we can easily show that

(i) If $u \in Q$, then $(A u)^{\prime \prime \prime}(t)=-f(t, u), t \in(0,1)$, hence $A u \in Q \cap C^{3}[(0,1), E]$.

(ii) If $u \in Q$ satisfies $A u=u$, then $u$ is a solution of the BVP (1.1)-(1.2).

Therefore, the BVP (1.1)- 1.2 is equivalent to the operator equation $A u=$ $u, u \in Q$.

Lemma 2.3. [15], [16] Let $D$ be a bounded set of $E$ and the map $f:[a, b] \times D \rightarrow E$ be bounded. Assume that $f(t, u)$ is uniformly continuous with respect to $t$. Then, we have

$$
\alpha(f([a, b] \times S))=\max _{t \in[a, b]} \alpha(f(t, S)), \quad S \subset D .
$$

Lemma 2.4. [15], [16] If $H \subset C[[a, b], E]$ is bounded and equicontinuous, then

$$
\alpha(H([a, b]))=\max _{t \in[a, b]} \alpha(H(t)),
$$

where $H([a, b])=\{u(t) \mid u \in H, t \in[a, b]\}$.

Lemma 2.5. Assume that $(H 1),(H 2),(H 9)$ hold. Then, operator $A: Q \cap T_{l} \rightarrow Q$ is a strict set contraction operator.

Proof. It is clear that $A$ is a bounded and continuous operator from $Q \cap T_{l}$ into $Q$. Since $f(t, u)$ is bounded and uniformly continuous in $t$ on $[a, b] \times T_{l}$ for any $l>0$, it follows from Lemma 2.3 and $(2.2)$ that

$$
\alpha(f([a, b] \times D))=\max _{t \in[a, b]} \alpha(f(t, D)) \leq L \alpha(D), D \subset P \cap T_{l} .
$$

Let $S \subset C[[a, b], E]$ be bounded. We know that $A(S) \subset C[[a, b], E]$ is bounded and equicontinuous, so, by Lemma 2.4 .

$$
\alpha(A(S))=\max _{t \in[a, b]} \alpha(A(S(t)))
$$


where $A(S(t))=\{(A u)(t) \mid u \in S\} \subset E$ ( $t$ is fixed). Using the formula

$$
\int_{0}^{1} y(t) d t \in \overline{c o}\{y(t) \mid t \in[0,1]\} \text { for } y \in C[[0,1], E]
$$

and observing Lemma 2.2 and (2.4), we find

$$
\begin{aligned}
\alpha(A(S(t))) & \left.=\alpha\left(\left\{\int_{0}^{1} G(t, s) f(s, u(s)) d s\right) \mid u \in S\right\}\right) \\
& \leq \alpha(\overline{c o}\{G(t, s) f(s, u(s)) \mid s \in[0,1], u \in S\}) \\
& \leq M_{1} \alpha(\{f(s, u(s)) \mid s \in[0,1], u \in S\}) \\
& \leq M_{1} \alpha\left(f([0,1] \times B) \leq M_{1} L \alpha(B), t \in[a, b],\right.
\end{aligned}
$$

where $B=\{u(s) \mid s \in[0,1], u \in S\}$. For any given $\varepsilon>0$, there is a partition $S=\bigcup_{j=1}^{n} S_{j}$ such that

$$
\operatorname{diam}\left(S_{j}\right)<\alpha(S)+\frac{\varepsilon}{3}, \quad j=1,2, \cdots, n .
$$

Choosing $u_{j} \in S_{j}(j=1, s, \cdots, n)$ and a partition of $J: a=t_{0}<t_{1}<\cdots<t_{m}=$ $b$ such that

$$
\left\|u_{j}(t)-u_{j}(s)\right\|<\frac{\varepsilon}{3}, \quad \forall j=1, s, \cdots, n ; t, s \in\left[t_{i-1}, t_{i}\right], i=1,2, \cdots, m .
$$

Obviously, $B=\bigcup_{i=1}^{m} \bigcup_{j=1}^{n} B_{i j}$, where $B_{i j}=\left\{u(s) \mid s \in\left[t_{i-1}, t_{i}\right], u \in S_{j}\right\}$. For any $u(t), \bar{u}(\bar{t}) \in B_{i j}\left(t, \bar{t} \in\left[t_{i-1}, t_{i}\right], u, \bar{u} \in S_{j}\right)$. It follows from 2.7), 2.8 that

$$
\begin{aligned}
\|u(t)-\bar{u}(\bar{t})\| & \leq\left\|u(t)-u_{j}(t)\right\|+\left\|u_{j}(t)-u_{j}(\bar{t})\right\|+\left\|u_{j}(\bar{t})-\bar{u}(\bar{t})\right\| \\
& \leq\left\|u-u_{j}\right\|_{C}+\frac{\varepsilon}{3}+\left\|u_{j}-\bar{u}\right\|_{C} \leq 2 \operatorname{diam}\left(S_{j}\right)+\frac{\varepsilon}{3}<2 \alpha(S)+\varepsilon .
\end{aligned}
$$

Consequently,

$$
\operatorname{diam}\left(B_{i j}\right) \leq 2 \alpha(S)+\varepsilon, \forall i=1,2, \cdots, m, j=1, s, \cdots, n,
$$

and so $\alpha(B) \leq 2 \alpha(S)+\varepsilon$, which implies, since $\varepsilon$ is arbitrary,

$$
\alpha(B) \leq 2 \alpha(S) .
$$

It follows then from $(2.5), 2.6,(2.9)$ that $\alpha(A(S)) \leq 2 M_{1} L \alpha(S), \quad S \subset Q \cap T_{l}$ with $2 M_{1} L<1$, and the Lemma 2.5 is proved.

We will apply the following fixed point theorem to obtain solutions of the BVP (1.1)-1.2).

Lemma 2.6. [16] Let $E$ be a Banach space, $K \subset E$ be a cone in $E, R>r>$ $0, K(r, R)=\{u \in K \mid r \leq\|u\| \leq R\}$, and let $A: K(r, R) \rightarrow K$ be a strict set contract operator such that either

(i) $A u \nsupseteq u, \forall u \in K,\|u\|=r$ and $A u \not \leq u, \forall u \in K,\|u\|=R$; or

(ii) $A u \not \leq u, \forall u \in K,\|u\|=r$ and $A u \nsupseteq u, \forall u \in K,\|u\|=R$.

Then $A$ has at least a fixed point in $K(r, R)$. 


\section{MAin RESUlTS}

Theorem 3.1. Let $P$ be a normal cone in E. Suppose that the conditions (H1)(H5) and (H9) are satisfied. Then the singular boundary value problem (1.1)(1.2) has at least two positive solutions.

Proof. Let

$$
K=\left\{u \in Q \mid u(t) \geq \gamma u(s), t \in\left[\frac{\eta}{\xi}, \eta\right], s \in[0,1]\right\},
$$

then $K$ is a cone in $C[[0,1], E]$ and satisfies $K \subset Q$.

We show that $A(K) \subset K$. In fact, for $u \in Q, t \in\left[\frac{\eta}{\xi}, \eta\right]$, by Lemma 2.2 we get

$$
\begin{aligned}
(A u)(t) & =\int_{0}^{1} G(t, s) f(s, u(s)) d s \geq \gamma \int_{0}^{1} \Phi(s) f(s, u(s)) d s \\
& =\gamma(A u)(x), \forall x \in[0,1] .
\end{aligned}
$$

This shows that $A u \in K$, hence $A(Q) \subset K$ and naturally $A(K) \subset K$.

By $(H 3)$ there exist a $r_{0} \in\left(0, \eta_{0}\right)$ and $\tau_{1}>0$ such that $u \in P,\|u\| \leq r_{0}$ implies

$$
\varphi(f(t, u)) \geq\left(m_{0}+\tau_{1}\right) \varphi(u), \quad t \in\left[\frac{\eta}{\xi}, \eta\right],
$$

where $\eta_{0}$ is as in $(H 5)$. We show that

$$
A u \not u, \quad \forall u \in K, \quad\|u\|_{C}=r_{0} .
$$

In fact, if there exists $u_{0} \in K,\left\|u_{0}\right\|_{c}=r_{0}$ such that $A u_{0} \leq u_{0}$, then, by the definition of $K$ and the normality of $P$ we have that

$$
N\left\|u_{0}(t)\right\| \geq \gamma\left\|u_{0}(s)\right\|, t \in\left[\frac{\eta}{\xi}, \eta\right], s \in[0,1],
$$

hence $\left\|u_{0}(t)\right\| \geq \frac{\gamma}{N}\left\|u_{0}\right\|_{C}=\frac{\gamma}{N} r_{0}, t \in\left[\frac{\eta}{\xi}, \eta\right]$. Taking

$$
\lambda=\min \left\{\varphi\left(u_{0}(t)\right) \mid t \in\left[\frac{\eta}{\xi}, \eta\right]\right\},
$$

then $\lambda>0$. On the other hand, for $t \in\left[\frac{\eta}{\xi}, \eta\right]$, we have that

$$
u_{0}(t) \geq\left(A u_{0}\right)(t) \geq \int_{\frac{\eta}{\xi}}^{\eta} G(t, s) f\left(s, u_{0}(s)\right) d s,
$$

so, by (3.1) we get, for $t \in\left[\frac{\eta}{\xi}, \eta\right]$,

$$
\begin{aligned}
\varphi\left(u_{0}(t)\right) & \geq \int_{\frac{\eta}{\xi}}^{\eta} G(t, s) \varphi\left(f\left(s, u_{0}(s)\right)\right) d s \geq\left(m_{0}+\tau_{1}\right) \int_{\frac{\eta}{\xi}}^{\eta} G(t, s) \varphi\left(u_{0}(s)\right) d s \\
& \geq\left(m_{0}+\tau_{1}\right) \lambda \int_{\frac{\eta}{\xi}}^{\eta} G(t, s) d s \geq \frac{m_{0}+\tau_{1}}{m_{0}} \lambda>\lambda .
\end{aligned}
$$

This is a contradiction to the definition of $\lambda$. Therefore, 3.2 holds.

By (H4) there exist $R_{0}>0$ and $\tau_{2}>0$ such that $u \in P,\|u\| \geq R_{0}$ implies

$$
\varphi_{1}(f(t, u)) \geq\left(m_{0}+\tau_{2}\right) \varphi_{1}(u), t \in\left[\frac{\eta}{\xi}, \eta\right] .
$$


Take $R>\max \left\{\frac{N R_{0}}{\gamma}, \eta_{0}\right\}$, where $\eta_{0}$ is as in $(H 5)$, then $R>\eta_{0}>r_{0}$. We show that

$$
A u \not z u, \quad \forall u \in K, \quad\|u\|_{C}=R .
$$

In fact, if there exists $u_{0} \in K,\left\|u_{0}\right\|_{C}=R$ such that $A u_{0} \leq u_{0}$, then, by the definition of $K$ and the normality of $P$ we have that

$$
N\left\|u_{0}(t)\right\| \geq \gamma\left\|u_{0}(s)\right\|, t \in\left[\frac{\eta}{\xi}, \eta\right], s \in[0,1] .
$$

Hence $\left\|u_{0}(t)\right\| \geq \frac{\gamma}{N}\left\|u_{0}\right\|_{C}=\frac{\gamma}{N} R>R_{0}, t \in\left[\frac{\eta}{\xi}, \eta\right]$. Taking

$$
\lambda_{1}=\min \left\{\varphi_{1}\left(u_{0}(t)\right) \mid t \in\left[\frac{\eta}{\xi}, \eta\right]\right\},
$$

then $\lambda_{1}>0$. On the other hand, for $t \in\left[\frac{\eta}{\xi}, \eta\right]$, we have that

$$
u_{0}(t) \geq\left(A u_{0}\right)(t) \geq \int_{\frac{\eta}{\xi}}^{\eta} G(t, s) f\left(s, u_{0}(s)\right) d s,
$$

so, by $(3.3)$ we get, for $t \in\left[\frac{\eta}{\xi}, \eta\right]$,

$$
\begin{aligned}
\varphi_{1}\left(u_{0}(t)\right) & \geq \int_{\frac{\eta}{\xi}}^{\eta} G(t, s) \varphi_{1}\left(f\left(s, u_{0}(s)\right)\right) d s \geq\left(m_{0}+\tau_{2}\right) \int_{\frac{\eta}{\xi}}^{\eta} G(t, s) \varphi_{1}\left(u_{0}(s)\right) d s \\
& \geq\left(m_{0}+\tau_{2}\right) \lambda_{1} \int_{\frac{\eta}{\xi}}^{\eta} G(t, s) d s \geq \frac{m_{0}+\tau_{2}}{m_{0}} \lambda_{1}>\lambda_{1} .
\end{aligned}
$$

This is a contradiction to the definition of $\lambda_{1}$. Therefore, (3.4) holds.

Now, we show that

$$
A u \nsupseteq u, \forall u \in Q,\|u\|_{C}=\eta_{0} .
$$

In fact, if there exists $u_{0} \in Q,\left\|u_{0}\right\|_{C}=\eta_{0}$ such that $A u_{0} \geq u_{0}$, then, by Lemma 2.2 and $(H 1)$ we know that

$$
\begin{aligned}
u_{0}(t) & \leq\left(A u_{0}\right)(t)=\int_{0}^{1} G(t, s) f\left(s, u_{0}(s)\right) d s \\
& \leq \int_{0}^{1} \Phi(s) g(s) h\left(u_{0}(s)\right) d s, t \in[0,1] .
\end{aligned}
$$

Hence, it follows from the normality of $P$ and $(H 5)$ that

$$
\begin{aligned}
\left\|u_{0}(t)\right\| & \leq N \int_{0}^{1} \Phi(s) g(s)\left\|h\left(u_{0}(s)\right)\right\| d s \\
& \leq N M_{0} \eta_{0} \int_{0}^{1} \Phi(s) g(s) d s, t \in[0,1] .
\end{aligned}
$$

Therefore, $\eta_{0}=\left\|u_{0}\right\|_{C}<\eta_{0} M_{0} N \int_{0}^{1} \Phi(s) g(s) d s=\eta_{0}$, which is a contradiction. Thus (3.5) holds.

By (3.2), 3.5), Lemmas 2.5 and 2.6 we know that the operator $A$ has at least one fixed point $u_{1}$ in $K\left(r_{0}, \eta_{0}\right)=\left\{u \in K \mid r_{0} \leq\|u\|_{C} \leq \eta_{0}\right\}$. Similarly, by (3.5), (3.4), Lemmas 2.5 and 2.6 we know that $A$ has at least one fixed point $u_{2}$ in $K\left(\eta_{0}, R\right)=\left\{u \in K \mid \eta_{0} \leq\|u\|_{C} \leq R\right\}$. Thus, the BVP (1.1)-(1.2) has at least two 
positive solutions $u_{1}(t)$ and $u_{2}(t)$ which satisfy $0<\left\|u_{1}\right\|_{C}<\left\|u_{2}\right\|_{C}$. Theorem 3.1 is proved.

Theorem 3.2. Let $P$ be a normal cone in E. Suppose that the conditions $(H 1),(H 2)$ and $(H 6)-(H 9)$ are satisfied. Then the singular boundary value problem (1.1)-1.2) has at least two positive solutions.

Proof. The proof is similar to Theorem 3.1 .

Example 3.3. Consider the following one dimensional singular boundary value problem

$$
\left\{\begin{array}{l}
u^{\prime \prime \prime}(t)+\frac{u^{2}}{2(1-t)^{\frac{3}{2}}}+\frac{\sqrt{u}}{16 t^{\frac{3}{2}}(1-t)^{\frac{3}{2}}}=0, \quad t \in(0,1), \\
u(0)=u^{\prime}(0)=0, u^{\prime}(1)=2 u^{\prime}\left(\frac{1}{4}\right)
\end{array}\right.
$$

Conclusion. The BVP (3.6) possesses at least two positive solutions $u_{1}(t)$ and $u_{2}(t)$ which satisfy $u_{i}(t)>0, t \in(0,1), i=1,2$.

Proof. Let $E=\mathbb{R}^{1}, P=[0, \infty)$, then $P$ is a normal cone in $E$. Hence

$$
f(t, u)=\frac{u^{2}}{2(1-t)^{\frac{3}{2}}}+\frac{\sqrt{u}}{16 t^{\frac{3}{2}}(1-t)^{\frac{3}{2}}},
$$

$f:(0,1) \times P \rightarrow P$ is continuous, the Green's function satisfies $G(t, s) \leq \Phi(s)=$ $6 s(1-s),(t, s) \in[0,1] \times[0,1]$.

It is clear that $f(t, u) \leq g(t) h(u)$, where $g(t)=\frac{1}{t^{\frac{3}{2}}(1-t)^{\frac{3}{2}}}, h(u)=\frac{u^{2}}{2}+\frac{\sqrt{u}}{16}$, and

$$
\int_{0}^{1} \Phi(s) g(s) d s=\int_{0}^{1} \frac{6}{\sqrt{s(1-s)}} d s=6 \pi .
$$

Hence, $(H 1)$ and $(H 2)$ are satisfied. Taking $\eta=\frac{1}{4}, \xi=2$, and $\varphi \in P^{*}, \varphi(u)=u$, we have that

$$
\lim _{u \rightarrow 0^{+}} \inf _{t \in\left[\frac{1}{8}, \frac{1}{4}\right]} \frac{\varphi(f(t, u))}{\varphi(u)}=\lim _{u \rightarrow 0^{+}} \inf _{t \in\left[\frac{1}{8}, \frac{1}{4}\right]}\left(\frac{u}{2(1-t)^{\frac{3}{2}}}+\frac{1}{16 t^{\frac{3}{2}}(1-t)^{\frac{3}{2}} \sqrt{u}}\right)=\infty,
$$

and

$$
\lim _{u \rightarrow \infty} \inf _{t \in\left[\frac{1}{8}, \frac{1}{4}\right]} \frac{\varphi(f(t, u))}{\varphi(u)}=\lim _{u \rightarrow \infty} \inf _{t \in\left[\frac{1}{8}, \frac{1}{4}\right]}\left(\frac{u}{2(1-t)^{\frac{3}{2}}}+\frac{1}{16 t^{\frac{3}{2}}(1-t)^{\frac{3}{2}} \sqrt{u}}\right)=\infty .
$$

Hence, (H3) and (H4) are satisfied. Moreover, since $N=1$ (the normal constant of $P$ ) and $M_{0}=\left(N \int_{0}^{1} \Phi(s) g(s) d s\right)^{-1}=\frac{1}{6}(\pi)^{-1}$, for $\eta_{0}=\frac{3}{2}$, it can follows easily that (H9) is satisfied. By Theorem 3.1, our conclusion follows. 


\section{REFERENCES}

1. M. Gregus, Third Order Linear Differential Equations, in: Math. Appl., Reidel, Dordrecht, 1987. 1]

2. G. Klaasen, Differential inequalities and existence theorems for second and third order boundary value problems, J. Diff. Equs. 10 (1971) 529-537.

3. L.K. Jackson, Existence and uniqueness of solutions of boundary value problems for third order differential equations, J. Diff. Equs. 13 (1993) 432-437.

4. D.J. ORegan, Topological transversality: Application to third order boundary value problems, SIAM J. Math. Anal. 19 (1987) 630-641.

5. A. Cabada, The method of lower and upper solutions for second, third, fourth and higher order boundary value problems, J. Math. Anal. Appl. 185 (1994) 302-320.

6. A. Cabada, The method of lower and upper solutions for third order periodic boundary value problems, J. Math. Anal. Appl. 195 (1995) 568-589.

7. A. Cabada, S. Lois, Existence of solution for discontinuous third order boundary value problems, J. Comput. Appl. Math. 110 (1999) 105-114.

8. A. Cabada, S. Heikkil'a, Extremality and comparison results for third order functional initial-boundary value problems, J. Math. Anal. Appl. 255 (2001) 195-212.

9. A. Cabada, S. Heikkil'a, Extremality and comparison results for discontinuous implicit third order functional initial-boundary value problems, Appl. Math. Comput. 140 (2003) 391-407.

10. Q. Yao, Solution and positive solution for a semilinear third-order two-point boundary value problem, Appl. Math. Lett. 17 (2004) 1171-1175.

11. Y. Sun, Existence of positive solutions for nonlinear third-order three-point boundary value problem, J. Math. Anal. Appl. 306 (2005) 589-603.

12. L. Guo, J. Sun, Y. Zhao, Existence of positive solutions for nonlinear third-order three-point boundary value problem, Nonlinear Anal. 68 (2008), 3151-3158. 1. 2.1. 2.2

13. K. Deimling, Ordinary differential equations in Banach spaces, LNM 886. New York, Berlin: Springer-Verlag, 1987. 1

14. V. Lakshmikantham and S. Leela, Nonlinear differential equations in abstract spaces, Pergamon, Oxford, 1981. 1

15. D. Guo, V. Lakshmikantham, Nonlinear Problems in Abstract Cones, Academic Press, Boston, MA, 1988. 2, 2.3, 2.4

16. D. Guo, V. Lakshmikantham and X. Liu, Nonlinear Integral Equations in Abstract Spaces, Kluwer Academic Publishers, Dordrecht, 1996. 2.3, 2.4, 2.6

1 School of Mathematics and Physics, Jiangsu Polytechnic University, Changzhou, 213164, PR CHINA.

E-mail address: fangzhang188@163.com 